\title{
Controversies in the Anesthetic Management of Lumbar Drains for Aortic Surgery
}

\section{Sarah Marie Cardillo and Wendy K Bernstein*}

Department of Anesthesiology, University of Maryland School of Medicine, Baltimore, Maryland, USA

*Corresponding author: Wendy K Bernstein, Department of Anesthesiology, University of Maryland School of Medicine, Baltimore, Maryland, USA, Tel: 410-328-6120; E-mail: wbernstein@anes.umm.edu

Rec date: Feb 13, 2015; Acc date: Feb 26, 2015; Pub date: Feb 28, 2015

Copyright: @ 2015 Cardillo SM, et al. This is an open-access article distributed under the terms of the Creative Commons Attribution License, which permits unrestricted use, distribution, and reproduction in any medium, provided the original author and source are credited.

\begin{abstract}
Lumbar drain insertion is an integral aspect of perioperative care for patients undergoing thoracoabodminal aortic aneurysm (TAA) repair. It has been shown to reduce incidence of paraplegia when both open and endovascular technique is used. Controversy exists in terms of optimal management of these catheters. This commentary will present current guidelines as well as experienced recommendations for their use.
\end{abstract}

Keywords: Lumbar drain; Thoracoabodminal aortic aneurysm; Cerebrospinal fluid

\section{Description}

Despite the increased use of endovascular techniques for thoracoabdominal aortic aneurysms (TAA), the risk of paraplegia after repair remains a significant concern for both the surgical and anesthesiology teams. Spinal cord ischemia or infarction with subsequent neurologic dysfunction occurs with an incidence of $4-13 \%$ after thoracic endovascular aortic repair (TEVAR) [1-3]. Decreased spinal cord perfusion persists postoperatively when episodes of hypotension, hemorrhage and elevated cerebrospinal fluid (CSF) pressure occur, which further increases likelihood of paraplegia after TEVAR [4]. Variation in both recommendations and practice continues to exist due to lack of consensus. Given the high acuity of these patients, the significant morbidity, and the necessity for optimal communication among all members of the perioperative care team, we present the recommendations based on current literature as well as commentary regarding anesthetic practices at our own institution, where a significant number of TEVAR cases are performed.

The greatest risk factors for paraplegia after TEVAR are the extent of endovascular stent coverage and prior distal aortic repair [5]. Increasing spinal cord perfusion through augmentation of arterial pressure, lumbar CSF drainage and reattachment of segmental arteries has been shown to reduce the incidence of paraplegia [6]. Spinal cord perfusion pressure is a function of lumbar CSF pressure subtracted from mean arterial pressure (MAP). Lumbar CSF drainage effectively improves spinal cord perfusion pressure via reduction of CSF pressure. Two separate meta analyses including 3 randomized controlled trials have demonstrated the efficacy of controlled drainage of CSF for prevention of neurological injury [6,7]. At our institution, we highly encourage the placement of lumbar drains for those patients at risk for paraplegia.

The placement of lumbar CSF drains is relatively safe and recommended for TEVAR. However, contraindications to placement include pre-existing coagulopathy, elevated intracranial pressure, and frequently, emergent surgery [8]. The procedure itself carries potential side effects, including intracranial hemorrhage ( $\mathrm{ICH}$ ) and neuraxial hematoma. The American Society of Regional Anesthesia and Pain Medicine (ASRA) advises that instrumentation of the neuraxis be avoided in patients with pre-existing coagulopathy, which may increase risk of bleeding with intrathecal catheter insertion [9]. This risk is elevated with recent or concurrent administration of antiplatelet agents or anticoagulants. In addition, guidelines state that the time from the procedure and anticoagulation dosing should exceed 60 minutes and that the lowest dose of heparin be administered [9].

CSF drainage can be performed prior to the operation in high risk patients or after the procedure if symptoms of spinal cord ischemia occur. The timing of placement varies by center. Benefits of preinduction placement include knowledge of the baseline CSF pressure, increased length of time prior to anticoagulation as well as knowledge of the patient's pain or paresthesis during placement, which could indicate potential nerve injury $[10,11]$. In patients pre-screened as high risk or difficult placement, it may be beneficial to place the lumbar drain well before the start of the case when alternative methods such as fluoroscopy can be utilized. Unfortunately, such management requires the patient to be hospitalized preoperatively, which increases cost. Furthermore, prolonged catheter insertion can also place the patient at increased risk for infection [11]. The majority of patients at our institution receive their lumbar drain at the time of surgery. For high risk patients, arrangements are made to have fluoroscopy available at the time of surgery to facilitate placement.

The drain should be placed at the L3-4 or L4-5 level in either the seated or lateral decubitus position. Positioning is at the discretion of the anesthesiologist, as both positions offer advantages. The seated position allows for easier palpation of spinous processes through a midline insertion, which avoids the more lateral epidural plexus. In addition, the improved lumbar flexion allows potentially more space for needle insertion. Thus, the risk for bloody tap is decreased [8]. On the other hand, the lateral decubitus position limits hydrostatic pressure of CSF and therefore the potential risk of over-draining CSF [8]. The drain should be inserted $8-10 \mathrm{~cm}$, however depths as great as $30 \mathrm{~cm}$ have been reported [12]. Although deeper insertion minimizes the risk of inadvertent removal, it may also contribute to paresthesias and possible nerve root injuries [8]. 
Should a bloody tap be encountered during placement, the appropriate response varies by institution. Traumatic instrumentation of the neuraxis has been associated with up to $50 \%$ of neuraxial hematomas $[8,9]$. The occurrence of neuraxial hematoma is rare. In a case series of 162 patients undergoing full heparinization for extracorporeal circulation, there were no spinal hematomas [13]. Another retrospective study, however, reported two spinal hematomas in sixty five patients undergoing TAA repair [10]. At ours and other institutions, if a bloody tap is encountered, the practice is to discontinue drainage and proceed with placement at another level. If bloody tap results a second time, case cancellation is considered after discussion with the operative team [10]. It has been suggested that the case be delayed up to 24 hours, however, based on ASRA guidelines this is only indicated for cases involving full heparinization for cardiopulmonary bypass, and otherwise there is no data supporting case cancellation $[8,9]$.

Animal models have demonstrated a decreased risk for ischemic injury when goal CSF pressure is below $10 \mathrm{mmHg}$ [6]. Studies finding CSF drainage to be ineffective limited the total CSF drained to $50 \mathrm{~mL}$ as well as allowing the CSF pressure to elevate above $10 \mathrm{mmHg}$ [6]. Continuous monitoring intraoperatively with intermittent drainage allows awareness of sudden wave form disruption and possible drain occlusion. It also avoids high volume or rate drainage and potential intracranial hypotension. Protocols for CSF drainage from the lumbar drain range from 10 to $20 \mathrm{~mL} /$ hour depending on patient condition [8]. However, at our institution, it is rare to have $>20 \mathrm{ml} /$ hour drained in neurologically intact patients.

Management postoperatively requires vigilance in serial neurologic examinations. In asymptomatic patients, management goals of CSF pressure should continue to be below $10 \mathrm{mmHg}$ with a rate limit of 10-15 mL/h. Patients with neurologic deficit may require drainage down to $5 \mathrm{mmHg}$ or as much as $20 \mathrm{~mL} /$ hour, which has been shown to improve symptoms $[8,10]$. At our institution, the catheter is opened to passive drainage for CSF pressures greater than $10 \mathrm{mmHg}$, and pressure is then reassessed after each $15 \mathrm{~mL}$ of CSF drained. Maximum CSF drainage rate is $20 \mathrm{~mL} /$ hour and up to $250 \mathrm{~mL} /$ day. If the pressure is persistently elevated after $150 \mathrm{~mL}$ are drained, the drain position is evaluated and the transducer re-zeroed. If the patient reveals any neurologic signs or symptoms, the catheter is drained to below $10 \mathrm{mmHg}$ coupled with blood pressure augmentation to a MAP $>90 \mathrm{mmHg}$.

Blood in the draining CSF is another issue that may be encountered. This may be an indication of an intracranial bleed, the risk of which is associated with excessive CSF drainage and development of intracranial hypotension $[14,15]$. Bloody drainage has been shown to be a poor marker of an epidural cord hematoma [10]. Should a change in neurologic status accompany blood within the drain, recommended workup includes immediate imaging $[8,10]$. At our institution, brain imaging is additionally recommended for asymptomatic persistent bloody CSF drainage lasting more than 4 hours.

Based on current literature, drainage should be discontinued after 2-3 days if there is no sign of spinal cord ischemia. After this period, a fibrotic response will have occurred around the catheter, which seals the puncture site. Clamping the drain prior to removal may lead to greater chance of CSF leak secondary to elevated CSF pressure [10]. However, our institution recommends that the drain be clamped for 12-24 hours and the patient monitored for neurologic symptoms prior to removal. If a CSF leak occurs, conservative measures such as bed rest, limiting head of bed elevation and hydration are recommended [10].

Lumbar catheters are usually removed if there is no sign of neurologic dysfunction or spinal cord ischemia. Removal of the lumbar drain should always be performed by an experienced practitioner who is familiar with the catheters. Pulling the catheter out slowly and steadily will reduce the risk of catheter fracture. Positioning in the lateral decubitus position with hip and back flexion increases the space between the vertebrae and spinous processes. This reduces the force required for removal, thus reducing the risk of fracturing the catheter [8]. If catheter breakage occurs, it is essential to perform a CT scan of the spine to determine the track and position of the retained fragment. The decision to remove the retained portion should be made on a case by case basis [16].

It is common in many institutions as well as our own to obtain an anticoagulation profile and platelet count prior to removal of the lumbar drain. For patients on a heparin infusion, it should be discontinued 2-4 hours prior to removal and not restarted for a full 12 hours afterward and coagulation status should be assessed. Indwelling catheters can be removed 2-4 hours after the previous subcutaneous heparin dose, and the next dose administered one hour after removal [9]. Although there is a paucity of evidence, ASRA guidelines do not require assessment of PTT with subcutaneous heparin administered twice or thrice daily. However, at our institution, anticoagulation profiles are recommended since thrice daily dosing may cause an elevation in PTT in some patients [9]. Lastly, the catheter can be removed 12 hours after the last dose of once daily low molecular weight heparin. Twice daily dosing of low molecular weight heparin should not be initiated until after catheter removal. Adequate surgical hemostasis and reversal of coagulopathy should always be assured [9]. Additionally, at our institution, it is common practice to obtain a platelet count prior to drain removal, with $100,000 / \mathrm{mcL}$ being the minimal adequate count. Recently, our institutional guidelines were changed to recommend discontinuation of both prophylactic and therapeutic heparin administration to patients with lumbar drains.

The optimal management of lumbar drain catheters for TEVAR lacks consensus and requires further evaluation. Given the number of studies revealing benefit and rare incidence of complications, the procedure will continue to be an integral aspect of perioperative care of these patients. Vigilance is required to evaluate the patient not only for neurologic sequelae of their operation, but for potentially catastrophic consequences of the indwelling drain. Through the use of education and awareness at all levels of care, reduction in paraplegia can be safely achieved.

\section{References}

1. Greenberg RK, Lu Q, Roselli EE, Svensson LG, Moon MC, et al. (2008) Contemporary analysis of descending thoracic and thoracoabdominal aneurysm repair: a comparison of endovascular and open techniques. Circulation 118: 808-817.

2. Xenos ES, Minion DJ, Davenport DL, Hamdallah O, Abedi NN, et al. (2009) Endovascular versus open repair for descending thoracic aortic rupture: institutional experience and meta-analysis. Eur J Cardiothorac Surg 35: 282-286.

3. Jonker FH, Trimarchi S, Verhagen HJ, Moll FL, Sumpio BE, et al. (2010) Meta-analysis of open versus endovascular repair for ruptured descending thoracic aortic aneurysm. J Vasc Surg 51: 1026-1032.

4. Safi HJ, Miller CC 3rd, Azizzadeh A, Iliopoulos DC (1997) Observations on delayed neurologic deficit after thoracoabdominal aortic aneurysm repair. J Vasc Surg 26: 616-622. 
Citation: Cardillo SM, Bernstein WK (2015) Controversies in the Anesthetic Management of Lumbar Drains for Aortic Surgery. J Vasc Med Surg

Page 3 of 3

5. Messé SR, Bavaria JE, Mullen M, Cheung AT, Davis R, et al. (2008) Neurologic outcomes from high risk descending thoracic and thoracoabdominal aortic operations in the era of endovascular repair. Neurocrit Care 9: 344-351.

6. Cina CS, Abouzahr L, Arena Go, , Laganà A, Devereaux PJ, et al. (2004) Cerebrospinal fluid drainage to prevent paraplegia during thoracic and thoracoabdominal aortic aneurysm surgery: a systematic review and meta-analysis. J Vasc Surg 40: 36-44.

7. Khan SN, Stansby G (2004) Cerebrospinal fluid drainage for thoracic and thoracoabdominal aortic aneurysm surgery. Cochrane Database Syst Rev.

8. Fedorow CA, Moon MC, Mutch WA, Grocott HP (2010) Lumbar cerebrospinal fluid drainage for thoracoabdominal aortic surgery: rationale and practical considerations for management. Anesth Analg 111: 46-58.

9. Horlocker TT, Wedel DJ, Rowlingson JC, Enneking FK, Kopp SL, et al. (2010) Regional anesthesia in the patient receiving antithrombotic or thrombolytic therapy: American Society of Regional Anesthesia and Pain Medicine Evidence-Based Guidelines (Third Edition). Reg Anesth Pain Med 35: 64-101.

10. Weaver K, Wiseman D, Farber M, Ewend M, Marston W, et al. (2001) Clinical Research Studies: Complications of lumbar drainage after thoracoabdominal aortic aneurysm repair. Journal of Vascular Surgery 34: 623-627.
11. Weigang E, Hartert M, Siegenthaler MP, Beckmann NA, Sircar R, et al. (2006) Perioperative management to improve neurologic outcome in thoracic or thoracoabdominal aortic stent-grafting. Ann Thorac Surg 82: 1679-1687.

12. Cheung A, Pochettino A, Guvakov D, Weiss S, Shanmugan S, et al. (2003) Safety of lumbar drains in thoracic aortic operations performed with extracorporeal circulation. The Annals Of Thoracic Surgery [serial online] 76: 1190-1197.

13. Estrera A, Sheinbaum R, Miller CC, Azizzadeh A, Walkes JC, et al. (2009) Original article: Cerebrospinal Fluid Drainage During Thoracic Aortic Repair: Safety and Current Management. The Annals of Thoracic Surgery [serial online]. 88: 9-15.

14. Vaughn SB, Lemaire SA, Collard CD (2011) Case scenario: anesthetic considerations for thoracoabdominal aortic aneurysm repair. Anesthesiology 115: 1093-1102.

15. Sinha AC, Cheung AT (2010) Spinal cord protection and thoracic aortic surgery. Curr Opin Anaesthesiol 23: 95-102.

16. Olivar H, Bramhall JS, Rozet I, Vavilala MS, Souter MJ, et al. (2007) Subarachnoid lumbar drains: a case series of fractured catheters and a near miss. Can J Anaesth 54: 829-834. 